\title{
CONHECIMENTO E RISCOS PARA ACIDENTE VASCULAR CEREBRAL EM MULHERES
}

KNOWLEDGE AND RISKS FOR STROKE IN WOMEN

CONOCIMIENTO Y RIESGOS PARA ACCIDENTE CEREBROVASCULAR EN MUJERES

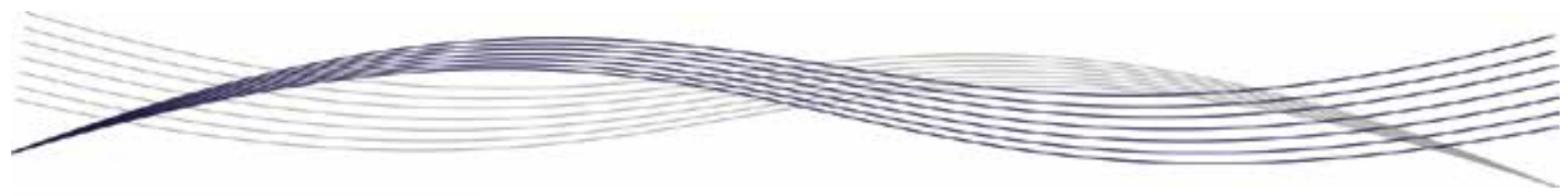

Palavras-chave:

Acidente Vascular Cerebral;

Enfermagem; Fatores de Risco.

Keywords:

Stroke; Nursing; Risk Factors.

Palabras clave:

Accidente Cerebrovascular; Enfermería; Factores de Riesgo.

Submetido: $17 / 05 / 2018$

Aprovado: $30 / 10 / 2018$

Autor(a) para Correspondência: Marcus Brenno Ferreira da Silva Avenida Dr. José Arimáteia Monte e Silva, $n^{\circ}$ 758, Apto. 102, Bloco A, Bairro: Campo dos Velhos, CEP.: 62030-233, Sobral-CE. E-mail:enf.brenno@gmail.com

\section{RESUMO}

Este artigo tem por objetivo averiguar o conhecimento e os fatores de risco para acidente vascular cerebral (AVC) em mulheres. Trata-se de pesquisa exploratória e descritiva, com abordagem qualitativa, realizada em setembro e outubro de 2014 com 55 mulheres em uma unidade de saúde em Ipueiras (CE). Os resultados indicam que os conhecimentos das mulheres tinham relação com os fatores de risco e os sinais e sintomas no desenvolvimento da doença, bem como com a falta de autocuidado e as preocupações do cotidiano. Elas reconheceram que se trata de uma doença grave, que debilita e pode levar à morte. Os fatores de risco observados foram sedentarismo, estresse, alimentação inadequada, etilismo, tabagismo, hipertensão arterial sistêmica (HAS) e obesidade. Constatouse a necessidade de adotar medidas voltadas à prevenção desses fatores de risco, principalmente dos modificáveis, em programas já instituídos na atenção primária à saúde (APS), o que aprimora o conhecimento da população acerca da doença e contribui com sua melhor qualidade de vida.

1. Enfermeira graduada pelo Instituto Superior de Teologia Aplicada (Inta). Sobral (CE), Brasil. E-mail: celianeamarante@hotmail.com

2. Enfermeiro. Aluno de Pós-Graduação em Enfermagem Cardiovascular no Instituto Lato Sensu. Sobral (CE), Brasil. E-mail: enf.brenno@gmail.com

3. Enfermeira. Doutora em Cuidados Clínicos pela Universidade Estadual do Ceará (UECE). Professora no Curso de Graduação em Enfermagem da Universidade Estadual Vale do Acaraú (UVA). Sobral (CE), Brasil. E-mail: keilinhaponte@hotmail.com 


\section{ABSTRACT}

This article aims to examine the knowledge on and the risk factors for stroke in women. This is an exploratory and descriptive research, with a qualitative approach, carried out in September and 0ctober 2014 with 55 women at a primary health service in Ipueiras, Ceará, Brazil. The results indicate that women's knowledge was related to risk factors and signs and symptoms in the course of disease, as well as lack of self-care and daily life concerns. They recognized that this is a serious disease, which makes people weak and can lead to death. The risk factors observed were sedentary lifestyle, stress, inadequate diet, alcohol consumption, smoking, hypertension, and obesity. The need to adopt measures aimed at the prevention of these risk factors was found, especially those modifiable, in programs already established in primary health care (PHC), and this improves the population's knowledge about disease and contributes to its better quality of life.

\section{RESUMEN}

Este artículo tiene como objetivo examinar el conocimiento y los factores de riesgo del accidente cerebrovascular $(A C V)$ en mujeres. Esta es una investigación exploratoria y descriptiva, con abordaje cualitativo, realizada en septiembre y octubre de 2014 con 55 mujeres en un servicio de atención primaria de salud en Ipueiras, Ceará, Brasil. Los resultados indican que los conocimientos de las mujeres estaban relacionados con los factores de riesgo y los signos y sintomas en el curso de la enfermedad, asi como con la falta de autocuidados y las preocupaciones de la vida cotidiana. Ellas reconocieron que esta es una enfermedad grave, que debilita a las personas y puede conducir a la muerte. Los factores de riesgo observados fueron sedentarismo, estrés, dieta inadecuada, consumo de alcohol, tabaquismo, hipertensión y obesidad. Se constató la necesidad de adoptar medidas dirigidas a la prevención de estos factores de riesgo, especialmente los modificables, en programas ya establecidos en la atención primaria de salud (APS), lo que mejora el conocimiento de la población sobre la enfermedad y contribuye a su mejor calidad de vida.

\section{INTRODUÇÃ O}

0 acidente vascular cerebral (AVC) é classificado como a terceira causa de morte entre adultos e idosos no Brasil. Na maioria dos casos, as pessoas acometidas se tornam dependentes de cuidados, muitos deles em domicílio. Essa doença tem crescido em virtude do aumento da expectativa de vida da população brasileira ${ }^{1-2}$.

0 AVC é caracterizado pela deficiência do estado neurológico, na maioria das vezes centralizado, de instalação súbita e repentina evolução de causa vascular. Tal circunstância vascular pode estar associada a alterações estruturais dos vasos funcionais, como as ligadas ao fluxo sanguíneo ou ao sistema de coagulação $0^{3}$.

Essa doença foi a causa mais frequente de morte entre mulheres com idades de 20 a 49 anos na cidade de São Paulo, segundo um estudo baseado em declarações de óbito: esteve presente em 8,5\% do total dessas declarações e dentre os 324 casos de doenças hipertensivas identificados, 156 tiveram como causa básica de morte a doença isquêmica do coração ou o $\mathrm{AVC}^{4}$.

Nesse sentido, vale destacar a atuação da equipe de enfermagem junto aos pacientes acometidos por AVC. 0 enfermeiro deve ter conhecimento especializado para atuar em tratamentos pautados por uma especialidade médica, com vistas a cumprir os requisitos de capacidade e destreza na assistência humanizada e integral à saúde. Em contrapartida, observa-se uma limitada oferta de cursos de especialização em Enfermagem em Neurologia e Neurocirurgia, o que compromete a composição das equipes de enfermagem responsáveis pelo tratamento de tais pacientes no Brasil ${ }^{5}$.

Considerando que essa doença acomete novas pessoas todos os dias ao redor do mundo, ela impacta a saúde pública em nível global, constituindo a principal causa de incapacidade neurológica ao comprometer funções motoras e cognitivas vitais. A população feminina é bastante afetada pelas consequências do AVC. Além da ocupação profissional fora de casa, as mulheres são as principais responsáveis pelas tarefas domésticas, muitas vezes exercidas exclusivamente por elas; essa fonte de estresse se associa ao fator idade e predispõe a maior acometimento de AVC após a menopausa, tornando a população feminina mais vulnerável a doenças cardiovasculares e cerebrovasculares. 
Atualmente, observa-se em serviços de emergência hospitalar e unidades de terapia intensiva (UTI) que o número de pacientes acometidos por essa doença tem aumentado. Assim, este estudo adotou a seguinte questão norteadora:

- Qual é o nível de conhecimento das mulheres sobre acidente vascular cerebral e quais são os riscos dessa doença na população feminina?

Tendo por objetivo averiguar o conhecimento e os fatores de risco para AVC em mulheres, este artigo se mostra relevante por indicar aos profissionais da saúde e à população geral a importância da qualidade de vida e da promoção da saúde para prevenir essa doença.

\section{METODOLOGIA}

Trata-se de pesquisa exploratória e descritiva, com abordagem qualitativa, realizada em setembro e outubro de 2014 na unidade de saúde "Casa da Mulher", localizada em Ipueiras (CE), a $300 \mathrm{~km} \mathrm{da}$ capital do estado (Fortaleza). Mantida pelo Sistema Único de Saúde (SUS), essa unidade de saúde foi criada em 2006 para atender somente à população feminina desse município, oferecendo consultas de enfermagem, consultas médicas e exames que enfocam desde a prevenção do câncer de colo do útero até análises mais avançadas, como ultrassonografia, entre outros recursos.

Foram convidadas a participar da pesquisa as usuárias da "Casa da Mulher" que se encontravam no local durante a coleta de dados. 0s critérios de inclusão adotados foram: mulheres com idades entre 18 e 60 anos; e usuárias que aguardavam o acolhimento na sala de espera. Já os critérios de exclusão foram: algum problema de saúde que dificultasse ou impossibilitasse o entendimento das questões; e queixa de dor variando de moderada a grave. Assim, 55 participantes foram incluídas nesta pesquisa.

Para a coleta de dados, desenvolveu-se um formulário para a caracterização dessas participantes: conhecimento sobre o AVC; potenciais causas dessa doença; estratégias de prevenção; histórico de conhecidos e familiares acometidos; e fatores de risco inerentes às mulheres.

Inicialmente, contatou-se a enfermeira responsável pela "Casa da Mulher", apresentando os objetivos e os aspectos da pesquisa. Em seguida, as mulheres elegíveis foram convidadas e confirmaram sua participação por meio da assinatura do termo de

\section{...essas mulheres são relativamente jovens em termos de risco para $A V C$.}

consentimento livre e esclarecido relativo ao estudo. Para preservar a identidade das participantes, elas fora identificadas pela letra “M" (Mulher), seguida pelo número sequencial de inclusão na pesquisa.

0 estudo obteve aprovação do Comitê de Ética em Pesquisa da Universidade Estadual do Ceará (UECE), sob o Parecer n. 789.919/2014, em conformidade com a Resolução CNS n. 466/2012 (Diretrizes e Normas Regulamentadoras de Pesquisas Envolvendo Seres Humanos).

As informações foram submetidas a análise de conteúdo temática, com as seguintes etapas:

1. Pré-análise - consiste em definir as unidades de registro, obtidas pela decomposição das falas ao destacar as palavras ou frases relevantes para o estudo;

2. Aplicação das categorias definidas análise dos trechos relevantes identificados nas falas;

3. Tratamento quantitativo - interpretação dos enunciados, com vistas a desvendar o conteúdo subjacente ( $p$. ex., ideologias e características dos fenômenos em questão) ${ }^{6}$.

\section{RESULTADOS E DISCUSSÃO}

Dentre as 55 participantes deste estudo, houve predomínio de mulheres entre 30 e 35 anos de idade $(36,36 \%)$, seguidas pelas faixas etárias entre 50 e $59 \operatorname{anos}(27,28 \%)$, entre 40 e 45 anos $(21,82 \%)$ e entre 18 e 25 anos (14,54\%). Pode-se dizer que essas mulheres são relativamente jovens em termos de risco para AVC.

$0 s$ dados levantados indicaram que $65,45 \%$ das participantes tinham filhos e $72,72 \%$ eram donas de casa. Houve predomínio de mulheres que se declararam pardas $(54,54 \%)$, seguidas por aquelas que se declararam brancas $(27,27 \%)$.

A seguir, apresentamos os demais resultados classificados em três categorias: a) Fatores de risco para AVC em mulheres; b) Conhecimento das 
mulheres sobre AVC; e c) Medidas de prevenção do AVC adotadas pelas mulheres.

\section{Fatores de risco para acidente vascular cerebral em mulheres}

0s fatores de risco predominantes foram: sedentarismo (20); estresse (19) e má alimentação (10). Também foram citados etilismo (7); tabagismo (5); hipertensão arterial sistêmica (HAS) (1); e obesidade (1). Mostra-se necessário adotar medidas voltadas à prevenção dos fatores de risco para AVC, principalmente os modificáveis (como sedentarismo, alimentação, etilismo e tabagismo).

0 perfil epidemiológico é marcado pela diferença no Brasil, no entanto, observa-se uma nova perspectiva no setor saúde, pois os indivíduos têm uma expectativa de vida cada vez maior. 0 envelhecimento populacional, aliado ao aumento dos índices de risco para doenças cerebrovasculares (como HAS, diabetes mellitus, tabagismo, etilismo, obesidade e dislipidemia), explica, em parte, a alta incidência de AVC no país ${ }^{8}$.

Dentre os fatores de risco para $A V C$ se destaca a idade avançada como mais frequente no sexo masculino. Quando o paciente já foi afetado por um AVC, a probabilidade de ter outro é maior e o histórico familiar também predispõe as pessoas a desenvolverem essa doença. HAS e diabetes mellitus ainda são grandes vilões, principalmente entre as pessoas sem tratamento adequado. Doenças cardíacas, tabagismo e abuso de álcool são outros fatores que contribuem com o AVC ${ }^{9}$.

As doenças crônicas não transmissiveis têm crescido significativamente nas últimas décadas, devido ao processo de globalização e à rápida urbanização, além do sedentarismo, da alimentação com alto teor calórico e do consumo de tabaco e álcool. Esses fatores de risco comportamentais causam impacto nos principais fatores de risco metabólicos (excesso de peso/obesidade, pressão arterial elevada, aumento da glicose sanguínea e do colesterol), o que resulta em doenças cardiovasculares e AVC, entre outras enfermidades ${ }^{9}$.

Os padrões de alimentação que a população adotou nas últimas décadas podem ser prejudiciais de diversas maneiras, por exemplo: o consumo exagerado de sal aumenta o risco de desenvolver HAS ou até uma doença cardiovascular. 0 alto consumo de carne vermelha, de carne altamente processada e de ácidos graxos se relaciona a doenças
As doenças crônicas

não transmissiveis têm crescido

significativamente nas últimas décadas...

cardiovasculares e diabetes mellitus. Por outro lado, o consumo regular de frutas e legumes é importante para diminuir o risco de doenças cardiovasculares, câncer de estômago e câncer colorretal ${ }^{10}$.

A população geral não adota medidas adequadas de prevenção. Há certa apreensão em relação às doenças, mas não uma ação efetiva para evitálas, como a prática de exercícios físicos, o hábito da alimentação saudável e o controle da pressão arterial.

Nesse contexto, mostra-se necessária a implementação de ações educativas, intervenções e campanhas, como as que observamos na prevenção do câncer de mama e do câncer de colo uterino, já estabelecidas dentro das unidades de saúde, proporcionando maior qualidade de vida às mulheres diante de adoecimento crônico não transmissivel auxiliando-as no enfrentamento de sua nova condição de existência, conduzindo-as ao pleno bem-estar e incentivando-as ao autocuidado ${ }^{11}$.

\section{Conhecimento das mulheres sobre acidente vascular cerebral}

Nesta categoria, aborda-se o conhecimento das mulheres sobre o AVC, bem como as estratégias de prevenção dos fatores de risco para essa doença. observou-se que o conhecimento delas tem relação com os sinais e sintomas da doença, assim como com os fatores de risco para o AVC.

É uma doença grave, é causada pela pressão alta. (M1)

É uma doença causada pelo anticoncepcional e estresse. (M2)

Acho que quem bebe muito, quem fuma, é mais fácil de ter essa doença. (M4)

Outras mulheres relataram que o desenvolvimento do AVC se deve à falta de autocuidado e às preocupações do cotidiano: 
Se dá por conta das pessoas não se cuidarem.

(M15)

Acredito que é uma doença causada devido à correria do dia a dia e pressão alta. (M12) Acredito que os hábitos de vida que temos influencia para termos essa doença. (M6)

Identificou-se nos discursos o reconhecimento dos sujeitos quanto ao quadro de sinais e sintomas do AVC, como hemiplegia, dormência e cefaleia:

É uma paralisação no corpo, uma dormência. (M33)

Acho que é uma forte dor de cabeça. (M19)

É grave! As pessoas que têm ficam com uma parte do corpo paralisada. (M29)

Elas também reconheceram que o AVC é uma doença grave que debilita e pode levar à morte:

É uma doença grave, debilita demais as pessoas. (M11)

É uma doença que é grave, acho que ninguém sobreviveu muito tempo. (M16)

É uma doença grave, quando dá acredito que não escapa, [há] poucas chances de sobreviver. (M24)

E associaram seus conhecimentos sobre a enfermidade aos saberes repassados de gerações em gerações por meio do senso comum. Vale ressaltar que muitas vezes esse saber não está em consonância com o conhecimento científico, o que torna necessária a participação de profissionais no processo da educação em saúde.

Abrir a geladeira com o corpo quente causa essa doença. (M38)

Comer comida quente e depois tomar banho. (M35)

0 AVC é classificado como a terceira causa de morte entre adultos e idosos, constituindo a principal causa de deficiência funcional e afetando, principalmente nos idosos, a eficiência de executar atividades cotidianas. Tais modificações podem ser passageiras ou não, de acordo com a região atingida, o nível da lesão e a capacidade individual de recuperação ${ }^{1}$.

0 sinal mais comum é a inconsciência, que pode
Apesar de existirem muitos casos de AVC em mulheres, nunca houve uma política de prevenção aplicada ao risco para AVC.

ocorrer de imediato após o sangramento na cavidade intracraniana, resultando na elevação da pressão intracraniana. Caso o paciente esteja consciente, ele terá cefaleia intensa e repentina combinada a hemorragia subaracnoidea. Também podemos citar como sinais e sintomas vômito, náuseas, convulsões, temperatura elevada, rigidez da nuca e sinais focais (como hemiparesia, afasia ou distúrbios visuais) ${ }^{12}$.

As deficiências neurológicas decorrentes do AVC variam conforme a região da lesão vascular, o tempo de perfusão inadequada e da existência de circulação colateral. Assim, esses eventos podem acarretar perda de força, de sensibilidade, de capacidade de movimentação e de controle de diversas áreas corporais, além de distúrbios de linguagem, perda do equilíbrio ou da coordenação e distúrbios visuais ${ }^{13}$.

Por conta dessas insuficiências funcionais, - AVC, além de diminuir a qualidade de vida das pacientes acometidas, causa grande impacto na vida dos familiares, ocasionando mudanças de rotina e reformulando o modo de vida das pessoas mais próximas. Nesse contexto, destaca-se que o conhecimento geral acerca da enfermidade, assim como de seus sinais e sintomas, tem contribuído para o prognóstico da doença ${ }^{14}$.

Apesar de existirem muitos casos de AVC em mulheres, nunca houve uma política de prevenção aplicada ao risco para AVC. As mulheres diferem dos homens em uma infinidade de aspectos, incluindo diferenças genéticas na imunidade e fatores hormonais, reprodutivos e sociais, aumentando o risco de AVC na população feminina ${ }^{13}$.

\section{Medidas de prevenção do acidente vascular cerebral adotadas pelas mulheres}

Nesta categoria, buscou-se levantar o conhecimento das participantes acerca de medidas que podem prevenir o AVC, além de investigar se elas adotam tais medidas em sua rotina diária. Destacamos as seguintes respostas: 
Não faço nada para prevenir. (M48)

Faço caminhada. (M42)

Procuro me alimentar [de modo] saudável. (M33)

A prática de exercícios físicos está associada a redução da morbimortalidade por diversas doenças, principalmente as cardiovasculares, levando ao aumento da expectativa de vida da população. Outros estudos têm demonstrado que a prática de exercícios físicos diminui com o aumento da idade, além de ser menor entre as mulheres e na população de baixa renda ${ }^{15}$.

Segundo um estudo, a população adulta apresenta baixos níveis de prática de exercícios físicos, equivalentes a $15 \%$ da amostra, e apenas $18,2 \%$ consomem 5 porções de frutas em 5 ou mais dias por semana, ao passo que $34 \%$ dos indivíduos consomem alimentos com elevado teor de gordura e $25 \%$ consomem refrigerante em 5 ou mais dias por semana. Tudo isso contribui com o aumento da prevalência do excesso de peso e, consequentemente, com a obesidade - que atinge cerca de $48 \%$ da população adulta ${ }^{16}$. Apesar da população temer as doenças cardiovasculares e cerebrovasculares, a maioria dos indivíduos não procura adotar hábitos mais saudáveis para preveni-las.

Nesse contexto, destacam-se os programas voltados à prática de exercícios físicos nas comunidades, que transcendem as ações assistencialistas oferecidas nos serviços de saúde por estimular as mudanças biopsicossociais, a promoção da saúde e a qualidade de vida. Tais programas não se limitam aos exercícios físicos no âmbito da população geral - envolvem orientação alimentar e saúde mental, promovendo a diminuição do estresse e a criação de vínculos ${ }^{17}$.

\section{CONCLUSÃO}

Ao averiguar o conhecimento e os riscos das mulheres para o AVC, constatou-se que os fatores de risco nessa população consistiram em sedentarismo, estresse, alimentação inadequada, etilismo, tabagismo, HAS e obesidade. Isso indica a necessidade de implementar medidas voltadas à prevenção desses fatores de risco, principalmente os modificáveis, bem como de aprimorar o conhecimento da população geral acerca dessa doença.

Quanto ao conhecimento das mulheres sobre AVC, observou-se que ele tem relação com os fatores de

\section{...a população adulta apresenta baixos níveis de prática de exercícios físicos...}

risco para desenvolver essa doença, além da falta de autocuidado e das preocupações do cotidiano. Elas reconhecem que se trata de uma doença grave que debilita e pode levar à morte, associando-a com seus sinais e sintomas (como hemiplegia, dormência e cefaleia) - o que equivale ao senso comum.

Houve certa dificuldade no local de pesquisa, pois alguns profissionais da unidade de saúde apresentaram resistência à presença da pesquisadora, dificultando a coleta de dados. Tendo em vista a limitada oferta de cursos de especialização em Enfermagem em Neurologia e Neurocirurgia, a composição das equipes de enfermagem responsáveis pelo tratamento de pacientes com AVC apresenta deficiências no Brasil. Isso corrobora a relevância deste artigo no contexto dos cuidados de enfermagem, que demandam 0 desenvolvimento de estratégias específicas voltadas à prevenção dessa doença em mulheres.

Porfim, apontamos a necessidade de novos estudos sobre o AVC em mulheres, pois a literatura dedicada a essa população é bem limitada. A prevalência dessa doença tem aumentado consideravelmente e a enfermagem precisa ampliar seu leque de recursos técnico-científicos para proporcionar um tratamento adequado às pacientes acometidas por ela.

\section{CONTRIBUIÇÃO DOS AUTORES}

Maria Celiane de Araújo contribuiu com a realização da pesquisa, o delineamento do estudo e a redação do manuscrito. Marcus Brenno Ferreira da Silva contribuiu com a revisão crítica do manuscrito. Keila Maria de Azevedo Ponte contribuiu com o delineamento do estudo e a redação e revisão crítica do manuscrito.

\section{REFERÊNCIAS}

1. Pedreira LC, Lopes RL. Cuidados domiciliares ao idoso que sofreu acidente vascular cerebral. Rev Bras Enferm [serial on the internet]. 2010 [cited 2014 Nov 25];63(5):837-40. Available from: http:// www.scielo.br/pdf/reben/v63n5/23.pdf 
2. Costa FAC, Silva DLA, Rocha VM. Estado neurológico e cognição de pacientes pós-acidente vascular cerebral. Rev Esc Enferm USP [serial on the internet]. 2011 [cited 2014 Sep 20];45(5):1083-8. Available from: http://www.scielo.br/pdf/reeusp/ v45n5/v45n5a08.pdf

3. Shettino G, Cardoso LF, Mattar Jr J, Ganem F, editors. Paciente crítico: diagnóstico e tratamento. Hospital Sírio-Libanês. 2. ed. Barueri (SP): Manole; 2012.

4. Chiavegatto Filho ADP, Laurenti R, Gotlieb SLD, Jorge MHPM. Mortalidade por doença hipertensiva em mulheres de 20 a 49 anos no Município de São Paulo, SP, Brasil. Rev Bras Epidemiol [serial on the internet]. 2004 [cited 2014 Sep 20];7(3):252-8. Available from: http://www.scielo.br/pdf/rbepid/ v7n3/03.pdf

5. Maniva SJC, Freitas CHA. Uso de alteplase no tratamento do acidente vascular encefálico isquêmico agudo: o que sabem os enfermeiros? Rev Bras Enferm [serial on the internet]. 2012 [cited 2014 0ct 28];65(3):474-81. Available from: http:// www.scielo.br/pdf/reben/v65n3/v65n3a12.pdf

6. Minayo MCS. 0 desafio do conhecimento: pesquisa qualitativa em saúde. 12. ed. São Paulo: Hucitec; 2010.

7. Cavalcante FT, Moreira PR, Guedes GN, Araújo LT, Lopes OVM, Damasceno CMM, et al. Intervenções de enfermagem aos pacientes com acidente vascular encefálico: uma revisão integrativa. Rev Esc Enferm USP [serial on the internet]. 2011 [cited 2014 Nov 29];45(6):1495-500. Available from: http://www. scielo.br/pdf/reeusp/v45n6/v45n6a31.pdf

8. Malta DC, Silva Jr JB. 0 plano de ações estratégicas para o enfrentamento das doenças crônicas não transmissiveis no Brasil e a definição das metas globais para o enfrentamento dessas doenças até 2025: uma revisão. Epidemiol Serv Saúde [serial on the internet]. 2013 [cited 2014 Nov 30];22(1):15164. Available from: http://scielo.iec.gov.br/pdf/ ess/v22n1/v22n1a16.pdf

9. Duncam BB, Chor D, Aquino EML, Bensenor IM, Mill GJ, Schinidt MI, et al. Doenças crônicas não transmissiveis no Brasil: prioridade para enfrentamento e investigação. Rev Saúde Pública [serial on the internet]. 2012 [cited 2014 Nov 20];46(Suppl):126-34. Available from: http://www. scielo.br/pdf/rsp/v46s1/17.pdf

10. Ponte KMA. Tecnologias do cuidado clínico de enfermagem para o conforto de mulheres com infarto agudo do miocárdio [dissertation]. Fortaleza: Universidade Estadual do Ceará; 2011 [cited 2018 Nov 5]. Available from: http://www.uece.br/cmacclis/ dmdocuments/keila_maria_de_azevedo_ponte.pdf. $p d f$
11. Barbieiri RLS. Os cuidados emergenciais. São Paulo: Rideel; 2010.

12. Lessmann JC, Conto F, Ramos G, Borenstein MS, Meirelles BHS. Atuação da enfermagem no autocuidado e reabilitação de pacientes que sofreram acidente vascular encefálico. Rev Bras Enferm [serial on the internet]. 2011 [cited 2014 0ct 10];64(1):198-202. Available from: http://www.scielo.br/pdf/reben/ v64n1/v64n1a30.pdf

13. Costa F, Oliveira $S$, Magalhães $P$, Costa B, Papini R, Silveira $M$, et al. Nível de conhecimento da população adulta sobre acidente vascular cerebral (AVC) em Pelotas - RS. J Bras Neurocir. 2008;19(1):31-7.

14. Ramalho JRO, Lopes ACS, Toledo MTT, Peixoto SV. Nivel de atividade física e fatores associados ao sedentarismo em usuários de uma unidade básica de saúde em Belo Horizonte, Minas Gerais. Rev Min Enferm [serial on the internet]. 2013 [cited 2014 0ct 29];18(2):426-32. Available from: www.reme. org.br/artigo/detalhes/937

15. Brasil. Estratégias de enfrentamento de doenças crônicas não transmissíveis. Brasília (DF): Ministério da Saúde; 2011.

16. Oliveira BN, Prado BL, Paiva GAM, Ribeiro MGS, Cardoso Junior OP. Análise qualitativa de um grupo de ginástica comunitária na atenção primária à saúde em Sobral-CE. Sanare (Sobral, Online) [serial on the internet]. 2013 [cited 20180 ct 27];12(2):6370. Available from: https://sanare.emnuvens.com. br/sanare/article/view/385/277
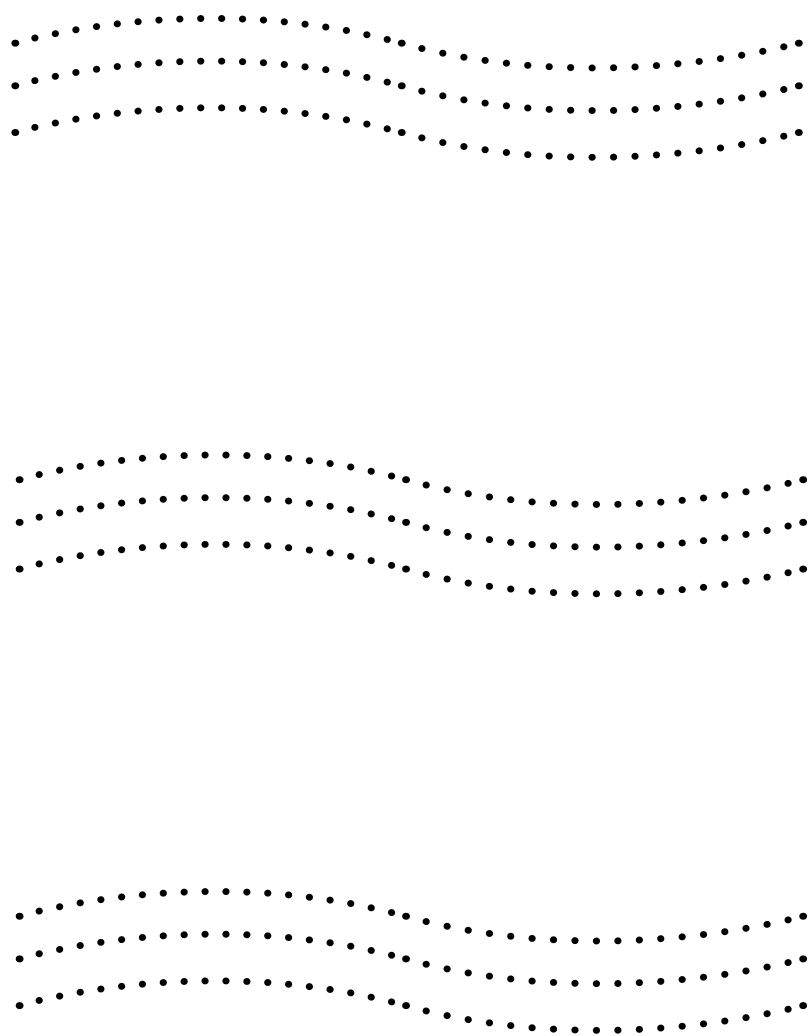